Global gyrokinetic simulations of TEM microturbulence

This article has been downloaded from IOPscience. Please scroll down to see the full text article.

2013 Plasma Phys. Control. Fusion 55074016

(http://iopscience.iop.org/0741-3335/55/7/074016)

View the table of contents for this issue, or go to the journal homepage for more

Download details:

IP Address: 128.178.125.186

The article was downloaded on 13/08/2013 at 13:39

Please note that terms and conditions apply. 


\title{
Global gyrokinetic simulations of TEM microturbulence
}

\author{
T Vernay ${ }^{1}$, S Brunner ${ }^{1}$, L Villard ${ }^{1}$, B F McMillan ${ }^{2}$, S Jolliet ${ }^{1}$, A Bottino ${ }^{3}$, \\ T Görler ${ }^{3}$ and F Jenko ${ }^{3}$ \\ ${ }^{1}$ Centre de Recherches en Physique des Plasmas, Ecole Polytechnique Fédérale de Lausanne (EPFL), \\ Association EURATOM-Confédération Suisse, CH-1015 Lausanne, Switzerland \\ ${ }^{2}$ Centre for Fusion, Space and Astrophysics, Department of Physics, University of Warwick, Coventry \\ CV4 7AL, UK \\ ${ }^{3}$ Max-Planck-Institut für Plasmaphysik, Boltzmannstrasse 2, EURATOM Association, D-85748 \\ Garching, Germany \\ E-mail: thibaut.vernay@epfl.ch
}

Received 18 October 2012, in final form 18 February 2013

Published 14 June 2013

Online at stacks.iop.org/PPCF/55/074016

\begin{abstract}
Global gyrokinetic simulations of electrostatic temperature-gradient-driven trapped-electron-mode (TEM) turbulence using the $\delta f$ particle-in-cell code ORB5 are presented. The electron response is either fully kinetic or hybrid, i.e. considering kinetic trapped and adiabatic passing electrons. A linear benchmark in the TEM regime against the Eulerian-based code GENE is presented. Two different methods for controlling the numerical noise, based, respectively, on a Krook operator and a so-called coarse-graining approach, are discussed and successfully compared. Both linear and non-linear studies are carried out for addressing the issue of finite- $\rho^{*}$-effects and finite electron collisionality on TEM turbulence. Electron collisions are found to damp TEMs through the detrapping process, while finite- $\rho^{*}$-effects turn out to be important in the non-linear regime but very small in the linear regime. Finally, the effects of zonal flows on TEM turbulence are briefly considered as well and shown to be unimportant in the temperature-gradient-driven TEM regime. Consistently, basically no difference is found between linear and non-linear critical electron temperature gradients in the TEM regime.
\end{abstract}

(Some figures may appear in colour only in the online journal)

\section{Introduction}

Microturbulence is widely believed to be responsible for anomalous transport in tokamak plasmas. Realistic tokamak parameters appear to lead to mixed ion-temperature-gradient (ITG) and trapped-electron-mode (TEM) turbulence. The adiabatic electron model, although very efficient from the practical point of view of computer resources, is not always applicable since the adiabatic assumption does not hold for trapped electrons nor for passing electrons located at low order mode rational flux surfaces (i.e. where the safety factor is rational: $q_{\mathrm{s}}=m / n, m$ and $n$ being integers) [1-3]. In particular, the adiabatic electron model does not allow the addressing of the electrostatic TEMs nor the electromagnetic instabilities. The hybrid electron model, briefly explained in this paper and designed for studying electrostatic TEM instabilities by providing a kinetic trapped electron response, was implemented quite early in the development of the ORB5 code, but only a few collisionless results have been published before this paper [4-6]. Note that the hybrid model has the main advantage of allowing larger time steps for TEM simulations compared with fully kinetic electron simulations, by a factor corresponding roughly to the ratio between the electron transit frequency $\omega_{\text {te }}$ and the electron bounce frequency $\omega_{\mathrm{be}}: \omega_{\mathrm{te}} / \omega_{\mathrm{be}}=1 / \sqrt{\epsilon}$, where $\epsilon=r / R_{0}$ is the local inverse aspect ratio. Moreover, the hybrid model prevents electrostatic shear Alfvén waves [7] from appearing and thus relaxes the corresponding constraint on the time step. Note that thorough and reliable TEM studies with a global gyrokinetic code, requiring the evolution of both kinetic ions and electrons, are very challenging computationally due to the multiple time and length scales involved in such multi-species 
simulations. As a consequence, very few global results have been published so far.

One of the major questions regarding the physics of TEM microturbulence is the importance of zonal flows in the turbulence saturation mechanism. The answer to this question remains complex and not fully understood. Non-linear global simulations of collisionless electrostatic TEM turbulence at $\eta_{\mathrm{e}}=\nabla \ln T_{\mathrm{e}} / \nabla \ln n_{\mathrm{e}} \simeq 3.1$ obtained using ORB5 are described in [6], emphasizing a moderate role of zonal flows in the saturation mechanism of non-linear temperature-gradientdriven TEM instabilities, in agreement with flux-tube (local) results obtained using the GENE code, see [8]. This latter result has recently been confirmed by other local simulations, stating that zonal flows only have an important saturation effect at $\eta_{\mathrm{e}} \lesssim 1$ [9]. Recent global simulations tend to show that zonal flows may constitute an important saturation mechanism in the case $T_{\mathrm{e}}=T_{\mathrm{i}}$ [10]. The importance of the parameter range in determining the efficiency of turbulence saturation by zonal flow shearing has been pointed out in [11] as well. For $\eta_{\mathrm{e}} \gtrsim 1$, an alternative TEM saturation mechanism through perpendicular particle diffusion is proposed in [12]. Note that a non-diffusive component in the electron heat flux due to TEM turbulence is obtained in [13].

In this paper, we first address some important numerical issues related to the $\delta f$ PIC algorithm applied to global collisional gyrokinetic TEM simulations. In particular, the relevance of the different noise-control procedures available for a PIC code, both in collisionless and collisional configurations, is discussed. Different physical cases are considered, featuring either a pure TEM spectrum or a TEMdominated spectrum. From a physical point of view, the role of zonal flows in TEM simulations is studied, as well as collisional effects and finite- $\rho^{*}$-effects on TEM turbulence.

This paper is organized as follows: section 2 presents the simulation model as well as the numerical methods used by ORB5, which have been shown to satisfy energy consistency [14]. Section 3 provides information about the physical systems that are considered, along with some code diagnostics. Section 4 shows results related to TEM benchmarks performed against the global Eulerian-based gyrokinetic code GENE [15]. Section 5 compares two different methods for controlling the numerical noise in PIC codes. Section 6 investigates the effects of plasma size on TEM dynamics. The effects of axisymmetric potential perturbations (i.e. zonal flows and geodesic acoustic modes) on TEM turbulence are briefly addressed in section 7 and further linked to TEM critical electron temperature gradient (ETG) in section 8. Results of simulations accounting for collisional electrons in the TEM regime are presented in section 9. Conclusions are drawn in section 10 .

\section{Simulation model and numerical methods}

\section{1. $\delta f$ gyrokinetic equation}

Simulations are performed with the global gyrokinetic code ORB5 $[16,17]$. The collisional model of ORB5 solves the gyrokinetic equation for the gyro-averaged ion distribution function $f_{\mathrm{i}}\left(\vec{R}, v_{\|}, \mu, t\right)$, where $\vec{R}$ is the gyrocentre position, $v_{\|}$is the parallel velocity and $\mu=m v_{\perp}^{2} / 2 B$ is the magnetic moment, along with the drift-kinetic equation for the electron distribution function $f_{\mathrm{e}}\left(\vec{R}, v_{\|}, \mu, t\right)$. A local Maxwellian background $f_{\mathrm{LM}}$ is chosen for both species. The ions are considered collisionless for TEM simulations, while the electron collision operator consists of a Lorentz operator for electron-ion collisions and a linearized Landau self-collision operator:

$\hat{C}\left(\delta f_{\mathrm{e}}\right)=C_{\mathrm{ei}}\left[\delta f_{\mathrm{e}}\right]+C_{\mathrm{ee}}\left[f_{\mathrm{LM}, \mathrm{e}}, \delta f_{\mathrm{e}}\right]+C_{\mathrm{ee}}\left[\delta f_{\mathrm{e}}, f_{\mathrm{LM}, \mathrm{e}}\right]$,

where $\delta f_{\mathrm{e}}=f_{\mathrm{e}}-f_{\mathrm{LM}, \mathrm{e}}$ represents the deviation of the full distribution $f_{\mathrm{e}}$ with respect to the local Maxwellian background $f_{\mathrm{LM}, \mathrm{e}}$. The first term on the right-hand side of equation (1) is the Lorentz operator, representing collisions of electrons on ions in the limit $m_{\mathrm{i}} \gg m_{\mathrm{e}}$. The second term on the right-hand side of equation (1) represents collisions of $\delta f_{\mathrm{e}}$ on the background $f_{\mathrm{LM}, \mathrm{e}}$ and the third term represents the background reaction (collisions of $f_{\mathrm{LM}, \mathrm{e}}$ on $\delta f_{\mathrm{e}}$ ) ensuring the local conservation of density, parallel momentum and kinetic energy. Dropping the electron index for simplicity, the approximated background reaction term is of the form $[18,19]$ :

$$
\begin{aligned}
& \frac{C\left[\delta f, f_{\mathrm{LM}}\right]}{f_{\mathrm{LM}}} \simeq \mathcal{B}(\delta f) \\
& \quad=\frac{1}{n_{0}}\left\{6 \sqrt{\pi} H(\mathrm{v}) \frac{\delta \mathcal{P}_{\|} v_{\|}}{v_{\mathrm{th}}^{2}}+\sqrt{\pi} G(\mathrm{v}) \frac{\delta \mathcal{E}}{v_{\mathrm{th}}^{2}}\right\},
\end{aligned}
$$

where $n_{0}$ is the background density, $v_{\text {th }}=\sqrt{T / m}$ is the thermal velocity and $\mathrm{v}=v / v_{\text {th }}$. The functions $H(\mathrm{v})$ and $G(\mathrm{v})$ are defined in [20] and related to the Rosenbluth potentials relative to a Maxwellian background. $\delta \mathcal{P}_{||}$and $\delta \mathcal{E}$ are respectively the changes in the parallel momentum and the kinetic energy of the fluctuation distribution due to $C\left[f_{\mathrm{LM}}, \delta f\right]$ :

$$
\begin{array}{r}
\delta \mathcal{P}_{\|}(\delta f, \vec{R})=-\int C\left[f_{\mathrm{LM}}, \delta f\right] v_{\|} \mathrm{d}^{3} v, \\
\delta \mathcal{E}(\delta f, \vec{R})=-\int C\left[f_{\mathrm{LM}}, \delta f\right] v^{2} \mathrm{~d}^{3} v .
\end{array}
$$

The form (2) ensures the same symmetry properties as the exact background reaction term and associated properties (H-theorem, conservation properties and stationary states).

A source term $S$ is also considered for both ions and electrons, such that the ion gyrokinetic equation and the electron drift-kinetic equation read, respectively:

$$
\begin{aligned}
\frac{\mathrm{D} \delta f_{\mathrm{i}}}{\mathrm{D} t} & =-\frac{\mathrm{D} f_{\mathrm{LM}, \mathrm{i}}}{\mathrm{D} t}+S\left(\delta f_{\mathrm{i}}\right), \\
\frac{\mathrm{D} \delta f_{\mathrm{e}}}{\mathrm{D} t} & +C_{\mathrm{ei}}\left[\delta f_{\mathrm{e}}\right]+C_{\mathrm{ee}}\left[f_{\mathrm{LM}, \mathrm{e}}, \delta f_{\mathrm{e}}\right] \\
& =-\frac{\mathrm{D} f_{\mathrm{LM}, \mathrm{e}}}{\mathrm{D} t}-C_{\mathrm{ee}}\left[\delta f_{\mathrm{e}}, f_{\mathrm{LM}, \mathrm{e}}\right]+S\left(\delta f_{\mathrm{e}}\right),
\end{aligned}
$$

where $\mathrm{D} / \mathrm{D} t$ is the collisionless gyrokinetic (respectively driftkinetic) operator:

$$
\frac{\mathrm{D}}{\mathrm{D} t}=\frac{\partial}{\partial t}+\frac{\mathrm{d} \vec{R}}{\mathrm{~d} t} \cdot \frac{\partial}{\partial \vec{R}}+\frac{\mathrm{d} v_{\|}}{\mathrm{d} t} \frac{\partial}{\partial v_{\|}} .
$$

The gyrokinetic (respectively drift-kinetic) equations of motion for the gyrocentre variables, derived by Hahm [21], 
are given by

$$
\begin{gathered}
\frac{\mathrm{d} \vec{R}}{\mathrm{~d} t}=\vec{v}_{\mathrm{G}}=v_{\|} \hat{b}+\underbrace{\vec{v}_{\nabla B}+\vec{v}_{\mathrm{c}}+\vec{v}_{E \times B},}_{\vec{v}_{\mathrm{d}}} \\
\frac{\mathrm{d} v_{\|}}{\mathrm{d} t}=\frac{1}{m v_{\|}} \vec{v}_{\mathrm{G}} \cdot(q \vec{E}-\mu \vec{\nabla} B), \\
\frac{\mathrm{d} \mu}{\mathrm{d} t}=0,
\end{gathered}
$$

which are valid for small fluctuation levels. Here $\hat{b}=\vec{B} / B$ is the unit vector along $\vec{B}, \vec{v}_{\mathrm{G}}$ is the gyrocentre velocity, $\vec{v}_{\nabla B}$ is the $\nabla B$ drift velocity, $\vec{v}_{\mathrm{c}}$ is the curvature drift velocity, $\vec{v}_{E \times B}$ is the $\vec{E} \times \vec{B}$ drift velocity and $\vec{E}$ is the gyro-averaged electric field deriving from the electrostatic potential $\phi$. Note that for electrons, the electric field at the gyrocentre position is considered instead of the gyro-averaged electric field (driftkinetic approximation).

\section{2. $\delta f$ particle-in-cell discretization}

ORB5 uses the low noise $\delta f$ particle-in-cell (PIC) method, requiring the introduction of $N$ numerical particles, called markers, for sampling the fluctuation distribution $\delta f$ of both species. Introducing two weights $w_{r}(t)$ and $p_{r}(t)$ for the marker $r$, the fluctuation distribution $\delta f$ and associated background distribution $f_{\mathrm{LM}}$, respectively, read:

$$
\begin{aligned}
& \delta f\left(\vec{R}, v_{\|}, \mu, t\right)=\sum_{r=1}^{N} \frac{m}{B_{\|}^{*}} w_{r}(t) \delta\left[\vec{R}-\vec{R}_{r}(t)\right] \\
& \quad \times \delta\left[v_{\|}-v_{\|, r}(t)\right] \delta\left[\mu-\mu_{r}(t)\right], \\
& f_{\mathrm{LM}}\left(\vec{R}, v_{\|}, \mu\right)=\sum_{r=1}^{N} \frac{m}{B_{\|}^{*}} p_{r}(t) \delta\left[\vec{R}-\vec{R}_{r}(t)\right] \\
& \quad \times \delta\left[v_{\|}-v_{\|, r}(t)\right] \delta\left[\mu-\mu_{r}(t)\right],
\end{aligned}
$$

where $B_{\|}^{*}=B+m v_{\|}(\vec{\nabla} \times \hat{b}) \cdot \hat{b} / q$ and $r$ is an index for labelling the $N$ markers. $\delta$ stands here for the Dirac function. The weight equations for each species are of the form

$$
\begin{gathered}
\frac{\mathrm{d}}{\mathrm{d} t} w_{r}(t)=-\left.p_{r}(t) \frac{1}{f_{\mathrm{LM}}} \frac{\mathrm{D}}{\mathrm{D} t} f_{\mathrm{LM}}\right|_{\left[z_{r}(t), t\right]} \\
-\left.p_{r}(t) \frac{C\left[\delta f, f_{\mathrm{LM}}\right]}{f_{\mathrm{LM}}}\right|_{\left[z_{r}(t), t\right]}, \\
\frac{\mathrm{d}}{\mathrm{d} t} p_{r}(t)=\left.p_{r}(t) \frac{1}{f_{\mathrm{LM}}} \frac{\mathrm{D}}{\mathrm{D} t} f_{\mathrm{LM}}\right|_{\left[z_{r}(t), t\right]},
\end{gathered}
$$

where the last term on the right-hand side of equation (13) is set to 0 for the collisionless ions, while in the case of electrons it represents the background reaction term of the self-collision operator $C_{\mathrm{ee}}\left[\delta f_{\mathrm{e}}, f_{\mathrm{LM}, \mathrm{e}}\right]$. Collisional and collisionless dynamics are separately considered through the time splitting approach. Solving the distribution equations for both species is thus performed by integrating numerically in time equations (8)-(10) to obtain the collisionless marker trajectories $z_{r}(t)=\left[\vec{R}_{r}(t), v_{\|, r}(t), \mu_{r}(t)\right]$ together with weight equations (13) and (14). The collisional marker trajectories for electrons, representing both $C_{\mathrm{ei}}\left[\delta f_{\mathrm{e}}\right]$ and $C_{\mathrm{ee}}\left[f_{\mathrm{LM}, \mathrm{e}}, \delta f_{\mathrm{e}}\right]$ in equation (6), are accounted for through random kicks in velocity space, as described in detail in [20].
Note that the linear runs are performed by dropping terms containing the perturbed electric field in the marker trajectories (equations (8)-(10)).

\subsection{Quasi-neutrality equation}

The electrostatic approximation is used in our model. Further considering the decomposition $f_{\mathrm{i}}=f_{\mathrm{LM}, \mathrm{i}}+\delta f_{\mathrm{i}}$, the background ion density $n_{\mathrm{i} 0}$ is given by the gyrodensity $\bar{n}_{\mathrm{i} 0}$ :

$$
\begin{aligned}
n_{\mathrm{i} 0} \simeq \bar{n}_{\mathrm{i} 0}(\vec{x})= & \int \frac{B_{\|}^{*}}{m} \mathrm{~d}^{3} R \mathrm{~d} v_{\|} \mathrm{d} \mu \mathrm{d} \alpha f_{\mathrm{LM}, \mathrm{i}}\left(\vec{R}, v_{\|}, \mu\right) \\
& \times \delta(\vec{R}+\vec{\rho}-\vec{x}),
\end{aligned}
$$

where $\alpha$ is the gyro-angle and $\vec{\rho}(\mu, \alpha)$ is the Larmor radius vector. One assumes that the background densities of electrons and ions verify quasi-neutrality, i.e. $Z \bar{n}_{\mathrm{i} 0}=n_{\mathrm{e} 0}$, where $Z$ is the ion charge. The perturbed ion density has both a perturbed gyrodensity contribution $\overline{\delta n}_{\mathrm{i}}$ as well as a polarization drift term $\delta n_{\text {pol,i }}$ :

$$
\delta n_{\mathrm{i}}(\vec{x}, t)=\overline{\delta n}_{\mathrm{i}}+\delta n_{\mathrm{pol}, \mathrm{i}} .
$$

In ORB5 the polarization drift term $\delta n_{\text {pol, } \mathrm{i}}$ is both linearized and expanded to second order in $k_{\perp} \rho_{\mathrm{Li}}$ (long wavelength approximation), where $k_{\perp}$ is the characteristic perpendicular wavenumber and $\rho_{\mathrm{Li}}$ the ion Larmor radius: $\delta n_{\mathrm{pol}, \mathrm{i}} \simeq \nabla_{\perp} \cdot\left[\left(n_{\mathrm{i} 0} / B \Omega_{\mathrm{i}}\right) \nabla_{\perp} \phi\right]$. Note that the polarization drift linearization assumes $|\delta f| \ll f_{\mathrm{LM}}$. In the case of driftkinetic electrons, the following quasi-neutrality equation for the fluctuating components is considered:

$-\nabla_{\perp} \cdot\left(\frac{n_{\mathrm{i} 0}(\Psi)}{B \Omega_{\mathrm{i}}} \nabla_{\perp} \phi(\vec{x}, t)\right)=\overline{\delta n}_{\mathrm{i}}(\vec{x}, t)-\frac{1}{Z} \delta n_{\mathrm{e}}(\vec{x}, t)$,

where $\delta n_{\mathrm{e}}$ is the perturbed electron density, $\Omega_{\mathrm{i}}$ is the ion cyclotron frequency and $\phi$ is the electrostatic potential. Since the electrons are considered drift-kinetic in equation (17), the electron density is in general equal to the electron gyrocentre density.

Within the frame of the so-called hybrid model, the kinetic electron response is computed over the trapped region of phase space, while the passing region is considered adiabatic. The quasi-neutrality equation thus becomes

$$
\begin{aligned}
& \left\langle\alpha_{\mathrm{p}}\right\rangle_{S}(\Psi) n_{\mathrm{i} 0} \frac{e\left[\phi-\langle\phi\rangle_{S}\right]}{T_{0 \mathrm{e}}(\Psi)}-\vec{\nabla}_{\perp} \cdot\left[\frac{n_{\mathrm{i} 0}}{B \Omega_{\mathrm{i}}} \vec{\nabla}_{\perp} \phi\right] \\
& =\overline{\delta n}_{\mathrm{i}}-\frac{1}{Z} \delta n_{\mathrm{e}}^{\text {trapped }},
\end{aligned}
$$

where $\left\langle\alpha_{\mathrm{p}}\right\rangle_{S}(\Psi)$ is the local flux-surface-averaged fraction of passing electrons and $\langle\phi\rangle_{S}$ is the flux-surface-averaged electrostatic potential. The adiabatic response of passing electrons is the first term on the left-hand side of equation (18), while the kinetic response of trapped electrons is the last term on the right-hand side of equation (18). Making use of a finite-element method, the quasi-neutrality equation is solved in straight-field-line magnetic coordinates $\left(s, \theta^{*}, \varphi\right)$, where $s$ is the flux surface label, $\theta^{*}$ is the straight-field-line poloidal angle and $\varphi$ is the toroidal angle. The considered grid size depends on the $\rho^{*}=\rho_{s} / a$ parameter, where $\rho_{s}$ is the sound Larmor radius and $a$ is the plasma minor radius. The number 
of grid points is typically $n_{s} \times n_{\theta^{*}} \times n_{\varphi}=128 \times 512 \times 256$ for $\rho^{*}=1 / 180$ and $n_{s} \times n_{\theta^{*}} \times n_{\varphi}=64 \times 256 \times 128$ for $\rho^{*}=1 / 80,1 / 60$. For simulations with fully kinetic electrons, $n_{s}$ is doubled.

\subsection{Sources and noise control}

The sources $S=S_{k}+S_{H}$ appearing in equations (5) and (6) consist of a Krook operator $S_{k}=-\gamma_{k} \delta f+S_{\text {corr }}$ and a heating operator

$S_{\mathrm{H}}=-\gamma_{\mathrm{H}}\left(\tilde{\delta f}(\Psi, \mathcal{E}, t)-\widetilde{f_{\mathrm{LM}}}(\Psi, \mathcal{E}, t) \frac{\int \mathrm{d} \mathcal{E} \tilde{\delta f}(\Psi, \mathcal{E}, t)}{\int \mathrm{d} \mathcal{E} \widetilde{f_{\mathrm{LM}}}(\Psi, \mathcal{E}, t)}\right)$,

the symbol ${ }^{\sim}$ standing for the operator which reconstructs the distribution in the $(\Psi, \mathcal{E})$ space, where $\Psi$ is the poloidal magnetic flux and $\mathcal{E}$ the kinetic energy. The details of the source terms are given in [22]. Note that the heat source is built in order to conserve the density and the zonal flows. Moreover, the Krook operator is built in order to conserve the density, parallel momentum and energy of the considered species, as well as the zonal flows, through the correction term $S_{\text {corr }}$.

The heat source $S_{\mathrm{H}}$ aims at maintaining an effective temperature profile $T=T(\Psi)$, both for ions and electrons. Transport processes indeed tend to relax the temperature profile while source terms tend to readjust it towards the reference background profile. The effective time-averaged profile thus equilibrates more or less near the reference background profile depending on the strength of the relaxation parameter $\gamma_{\mathrm{H}}$ in the source term. The Krook operator $S_{k}$ acts as a noise-control operator by applying a small decay of the weights, which allows us to carry out long turbulence simulations with a PIC code such as ORB5. Note that the Krook operator is systematically used in this paper to control the numerical noise associated with the collisionless ions. Typically $100 \times 10^{6}$ markers are used along with the Krook operator. Successful non-linear benchmarks between GENE and ORB5 have already been performed in the ITG regime $[23,24]$, providing a stringent test for the Krook operator as a noise-control procedure.

The Krook operator is, however, impractical for carrying out collisional simulations, since the required numerical decay rate $\gamma_{k}$ which needs to be chosen for the approach to be effective is typically of the order of the collision frequency and may thus significantly interfere with the corresponding physical effects. The control of numerical noise in the presence of collisions is thus handled by making use of the so-called coarse-graining procedure, first proposed in [19] and further simplified in [25]. The implementation of the coarse-graining procedure in the ORB5 code is described in [26]. The idea is to perform a binning of the markers in the $5 \mathrm{D}$ gyrokinetic phase space. Periodically (every $n_{\mathrm{cg}}$ th time step), the weight of each marker is set to the average weight value of all the markers within the same bin. The size of the bins needs to be carefully chosen, in order to prevent any artificial damping at relevant scales from occurring, both in configuration and velocity space. In configuration space, the number of bins is typically chosen according to the grid for the field solver, accounting for one major difference: in contrast to the field solver, the binning is performed in the field-aligned coordinates $\left(s, \theta^{*}, z=\varphi-q_{s} \theta^{*}\right), q_{s}$ being the safety factor. These coordinates allow us to take larger bins in the poloidal direction (typically $N_{s} \times N_{\theta^{*}} \times N_{z}=64 \times 32 \times 128$ for $\rho^{*}=1 / 60$ ), thus improving the statistics of the coarse-graining procedure. In velocity space, the binning is performed in the energy $(E)$-pitch angle $(\xi)$ space with a number of bins $n_{E} \times n_{\xi} \sim 64 \times 64$. The typical number of bins requires more electron markers than the Krook operator in order to control the noise properly, about $300 \times 10^{6}-600 \times 10^{6}$ markers.

\section{Equilibrium profiles and physical cases}

The considered ad hoc equilibrium [27] consists of a tokamak configuration with circular and concentric magnetic surfaces. In this case, the axisymmetric magnetic field is given by $\vec{B}=\vec{\nabla} \Psi \times \vec{\nabla} \varphi+F(\Psi) \vec{\nabla} \varphi$, assuming $\Psi=\Psi(r)$ with $\mathrm{d} \Psi / \mathrm{d} r=r B_{0} / \bar{q}(r)$, as well as $F=R B_{\varphi}=R_{0} B_{0}$, so that

$$
\vec{B}=\frac{B_{0} R_{0}}{R}\left(\hat{e}_{\varphi}+\frac{r}{R_{0} \bar{q}(r)} \hat{e}_{\theta}\right),
$$

where $R$ is the major radius, $r$ is the local minor radius, $\varphi$ is the toroidal angle, $\theta$ is the poloidal angle and $\hat{e}_{\varphi}, \hat{e}_{\theta}$ are the unit vectors in the toroidal and poloidal directions, respectively. Furthermore, $B_{0}$ and $R_{0}$ stand for the magnetic field amplitude and major radius on the magnetic axis $(r=0)$. The $\bar{q}$ profile is chosen quadratic: $\bar{q}(r)=\bar{q}_{0}+\bar{q}_{1} r^{2} / a^{2}$ and is related to the safety factor profile $q_{s}(r)$ by the relation $\bar{q}(r)=\sqrt{1-\epsilon^{2}} q_{s}(r)$. The values $\bar{q}_{0}=0.854$ and $\bar{q}_{1}=2.184$ are chosen such that $\bar{q}(r / a=0.5)=1.4$ and the magnetic shear $\left(r / q_{s}\right) \mathrm{d} q_{s} /\left.\mathrm{d} r\right|_{r=0.5 a}=\hat{s}(r / a=0.5) \simeq 0.75$.

Let $\mathcal{A}$ stand for either the temperature $T$ or density $n$ of a given species, and $\kappa_{\mathcal{A}}=a / L_{\mathcal{A}}$, where $L_{\mathcal{A}}$ is the characteristic length of the considered gradient. For non-linear simulations, profiles are defined with respect to the coordinate $r$, with a flat logarithmic gradient:

$$
\begin{gathered}
\frac{\mathrm{d} \ln \mathcal{A}}{\mathrm{d}(r / a)}=-\frac{\kappa_{\mathcal{A}}}{2}\left[\tanh \left(\frac{r-\left(r_{0}-\Delta_{\mathcal{A}}\right)}{\Delta_{r}}\right)\right. \\
\left.-\tanh \left(\frac{r-\left(r_{0}+\Delta_{\mathcal{A}}\right)}{\Delta_{r}}\right)\right],
\end{gathered}
$$

where the values $r_{0}=0.5 a$ for the centre of the gradient profile, $\Delta_{r}=0.04 a$ for the width of the ramps and $\Delta_{\mathcal{A}}=0.3 a$ for the gradient profile width are chosen. For linear simulations, peaked shape profiles are used:

$$
\frac{\mathrm{d} \ln \mathcal{A}}{\mathrm{d}(r / a)}=-\kappa_{\mathcal{A}} \cosh ^{-2}\left(\frac{r-\tilde{r}_{0}}{\Delta_{\mathcal{A}}}\right),
$$

with $\tilde{r}_{0}=0.5 a$ and $\Delta_{\mathcal{A}}=0.2 a$ or $\Delta_{\mathcal{A}}=0.3 a$.

The particle flux $\Gamma$ and the energy flux $Q$ are given by

$$
\begin{gathered}
\Gamma=\left\langle\int \delta f \vec{v}_{E \times B} \cdot \vec{\nabla} r \mathrm{~d}^{3} v\right\rangle_{S}, \\
Q=\left\langle\int \delta f \frac{m v^{2}}{2} \vec{v}_{E \times B} \cdot \vec{\nabla} r \mathrm{~d}^{3} v\right\rangle_{S},
\end{gathered}
$$


where \langle\rangle$_{S}$ stands for the flux-surface-average operator. The particle diffusivity is $D=\Gamma /|\nabla n|$, while the energy diffusivity is defined by $\chi^{E}=Q / n|\nabla T|$. Diffusivities are normalized with respect to the gyro-Bohm units $\rho_{s}^{2} c_{s} / a$, where $c_{s}=$ $\sqrt{Z T_{\mathrm{e}} / m_{\mathrm{i}}}$ is the sound speed evaluated at $r / a=0.5$ and $\rho_{s}=$ $c_{s} / \Omega_{\mathrm{i}}$ is the sound Larmor radius evaluated at $r / a=0.5$ with the magnetic field $B_{0}$ on axis. The zonal flow $\vec{E} \times \vec{B}$ shearing rate $\omega_{E \times B}$ is defined as the radial derivative of the radial electric field $E_{r}$ as follows: $\omega_{E \times B}=\left(r / q_{s} B_{0}\right) \mathrm{d} / \mathrm{d} r\left(q_{s} E_{r} / r\right)$ [28], where $E_{r}=-\mathrm{d}\langle\phi\rangle_{S} / \mathrm{d} r$.

The standard CYCLONE case [29] features dominant ITG modes at low $k_{\theta} \rho_{L \mathrm{i}}$ and dominant TEM modes at higher $k_{\theta} \rho_{L \mathrm{i}} \sim 1$. This physical situation is a major impediment to CYCLONE TEM studies with ORB5, since the quasineutrality solver is only valid for $k_{\theta} \rho_{L i} \lesssim 1$. In order to study a typical temperature-gradient-driven TEM instability, we define a TEM CYCLONE case, which is the CYCLONE case $\left(\rho^{*}=\rho_{s} / a=1 / 180\right.$, deuterium as ion species) with a weaker ion temperature gradient: $R_{0} / L_{T \mathrm{i}}=R_{0} / L_{n}=2.2$, $R_{0} / L_{T \mathrm{e}}=6.9$. Here, $L_{n}$ and $L_{T}$ are the characteristic lengths of the density and temperature gradients respectively. A smaller case, called the TEM TCV case, is built according to the parameters of the tokamak à configuration variable $(T C V)$. The TEM TCV case considers the same temperature, density and safety factor profiles as the TEM CYCLONE case and keeps the parameters $a / L_{n}=a / L_{T_{\mathrm{i}}} \simeq 0.8$ and $a / L_{T_{\mathrm{e}}} \simeq 2.5$ from the TEM CYCLONE case, along with the geometrical parameters $R_{0}=0.88 \mathrm{~m}, a=0.25 \mathrm{~m}$, $B_{0}=1.43 \mathrm{~T}$ and $\rho^{*}=1 / 80$. Moreover, deuterium is also chosen as the ion species. Choosing the parameters of either the TEM CYCLONE case or the TEM TCV case leads to dominant TEM instabilities even at low $k_{\theta} \rho_{L \mathrm{i}}$. Considering some TCV-relevant temperature and density values $(n \sim$ $10^{19}-2 \times 10^{19} \mathrm{~m}^{-3}$ and $T \sim 1-2.5 \mathrm{keV}$ ) provides a range of physical collisionality $v_{e i \text {,phys }} \sim 5 \times 10^{-3}-5 \times 10^{-2}\left[c_{s} / a\right]$. The same range of physical collisionality is obtained for the TEM CYCLONE case, considering relevant DIII-D parameters $\left(n \sim 2 \times 10^{19}-3 \times 10^{19} \mathrm{~m}^{-3}\right.$ and $\left.T \sim 2-5 \mathrm{keV}\right)$. For both cases, the conditions $T_{\mathrm{i}}=T_{\mathrm{e}}$ as well as $T_{\mathrm{i}}<T_{\mathrm{e}}$ are studied.

Note that neither TEM TCV nor TEM CYCLONE cases exhibit a purely temperature-gradient-driven TEM spectrum. Indeed, ETG modes are present at smaller poloidal wavelengths $k_{\theta} \rho_{L \mathrm{i}} \gtrsim 1$, which are not resolved both due to the assumption $k_{\theta} \rho_{L i}<1$ of the ORB5 solver and the fact that passing electrons are forced to respond adiabatically in the hybrid electron model used in ORB5, while ETG modes require a kinetic representation for this group of particles. A physical case considered only in section 7, exhibiting a socalled purely temperature-gradient-driven TEM spectrum, i.e. featuring no ETG modes nor density-gradient-driven TEMs, was identified through a parameter scan in the linear regime using the GENE code. This case is characterized by the following physical parameters: CYCLONE case geometry with $T_{\mathrm{i}} / T_{\mathrm{e}}=0.3, R_{0} / L_{n}=1.0, R_{0} / L_{T \mathrm{e}}=4.75$, and $R_{0} / L_{T \mathrm{i}}=3.0$. The corresponding linear spectrum is unstable in the range $0<k_{\theta} \rho_{L \mathrm{i}} \lesssim 0.6$.

Except for the results of section 4 where fully kinetic electrons are used, all other results are obtained within the frame of the hybrid electron model already introduced. Heavy electrons are usually considered $\left(m_{\mathrm{i}} / m_{\mathrm{e}}=200\right)$ in order to further facilitate the global simulations presented here. Note that using heavy electrons should be relevant as long as $\omega / \omega_{\text {be }} \ll 1$, where $\omega$ is the characteristic TEM frequency of the considered system and $\omega_{\text {be }}=\sqrt{\epsilon T_{\mathrm{e}} / m_{\mathrm{e}}} / q_{s} R_{0}$ is the electron bounce frequency. The time step chosen for TEM simulations performed through the hybrid model is typically $\Delta t \sim 2 \times 10^{-2}\left[a / c_{s}\right]$. Actually, a mass ratio $m_{\mathrm{i}} / m_{\mathrm{e}}=200$ has been checked to provide converged linear growth rates (within less than 5\%) in the frame of TEM simulations using the hybrid model described in this paper (see for instance [6], or figure 3.9 in [30]). Regarding non-linear TEM simulations in the frame of a fully kinetic electron model, a mass ratio $m_{\mathrm{i}} / m_{\mathrm{e}}=400$ was found to provide electron heat fluxes smaller by $\sim 25 \%$ compared with fluxes obtained with the realistic mass ratio [8]. However, the convergence with electron mass is clearly faster within the frame of the hybrid model and there is no significant difference expected in the turbulence level between a simulation with realistic $m_{\mathrm{i}} / m_{\mathrm{e}}=1836$ hybrid electrons and heavy $m_{\mathrm{i}} / m_{\mathrm{e}}=200$ hybrid electrons. Using heavy electrons is therefore an approximation but the hybrid model is probably a more significant limitation of the electron model.

\section{ORB5-GENE linear TEM benchmark}

Considering the TEM CYCLONE case with $T_{\mathrm{i}}=T_{\mathrm{e}}$ and the peaked gradient profiles for density and temperature, as described by equation (22) with $\Delta_{\mathcal{A}}=0.3 a$, a global collisionless linear benchmark in the TEM regime is performed against the global version of the Eulerian-based gyrokinetic code GENE [15]. In contrast to the other results and simulations presented in this paper, fully kinetic electrons of mass $m_{\mathrm{i}} / m_{\mathrm{e}}=400$ are considered in this section. In the electrostatic approximation, fully kinetic electrons may destabilize electrostatic shear Alfvén modes and thus require small time steps for proper TEM simulations with particles, as explained in [7]. The required time step scales with $\Delta t \sim k_{\theta}$ and thus needs to be even smaller for simulations considering long poloidal wavelengths. ORB5 uses time steps in the range $\Delta t \sim 5 \times 10^{-4}-5 \times 10^{-3}\left[a / c_{s}\right]$ for the considered TEM CYCLONE case with fully kinetic electrons. Due to the above-mentioned numerical challenges, the simulation for the lowest considered value $k_{\theta} \rho_{s}=0.125$ has in fact been performed in the case of ORB5 within the frame of the hybrid model. Figure 1 shows the benchmark results for both the growth rate spectrum and the real frequency spectrum. Note the very good agreement at long wavelengths $\left(k_{\theta} \rho_{s} \lesssim 0.5\right)$ between both codes. Discrepancies at larger $k_{\theta} \rho_{s} \gtrsim 0.5$ can probably be attributed to the long wavelength approximation assumed by the ORB5 field solver. The modes $k_{\theta} \rho_{s} \gtrsim 0.5$ are in fact not expected to contribute significantly to turbulent heat transport for the considered parameters [31] and the long wavelength approximation in the quasi-neutrality equation should therefore be an appropriate model for the corresponding turbulence simulations presented in this paper. In addition, local (flux-tube) results obtained with GENE are shown in 

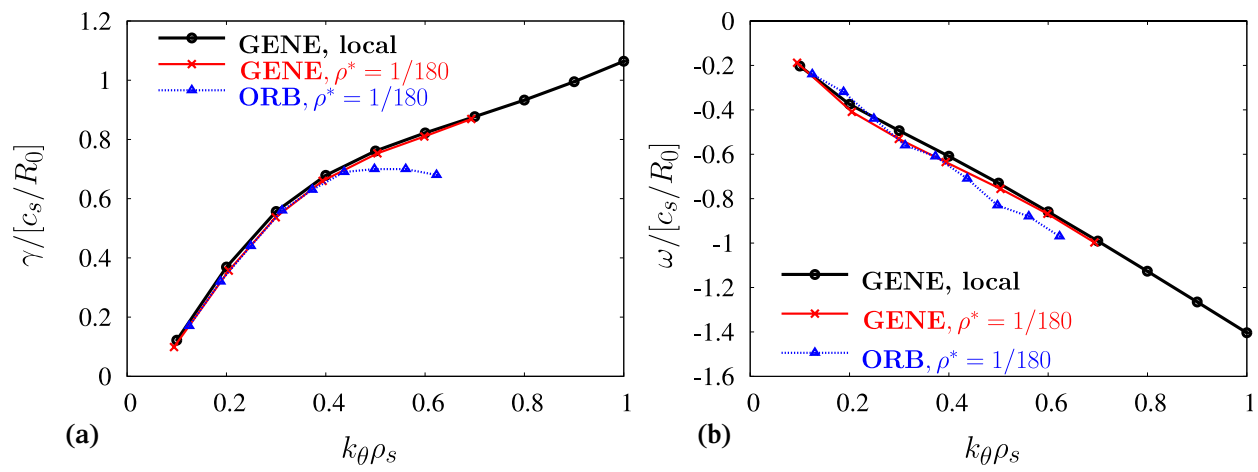

Figure 1. TEM CYCLONE case, $T_{\mathrm{e}}=T_{\mathrm{i}}$. Global simulations for $\rho^{*}=1 / 180$ and fully kinetic electrons (except $k_{\theta} \rho_{s}=0.125$ for ORB5 where hybrid electrons are considered). Linear benchmark in the TEM regime between ORB5 and GENE, providing good agreement regarding $(a)$ the linear growth rate spectrum and $(b)$ the real frequency spectrum for $k_{\theta} \rho_{s} \lesssim 0.5$. Discrepancies at larger $k_{\theta} \rho_{s} \gtrsim 0.5$ come from the long wavelength approximation assumed by the ORB5 field solver. For comparison, local (flux-tube) simulations obtained with GENE are also shown.
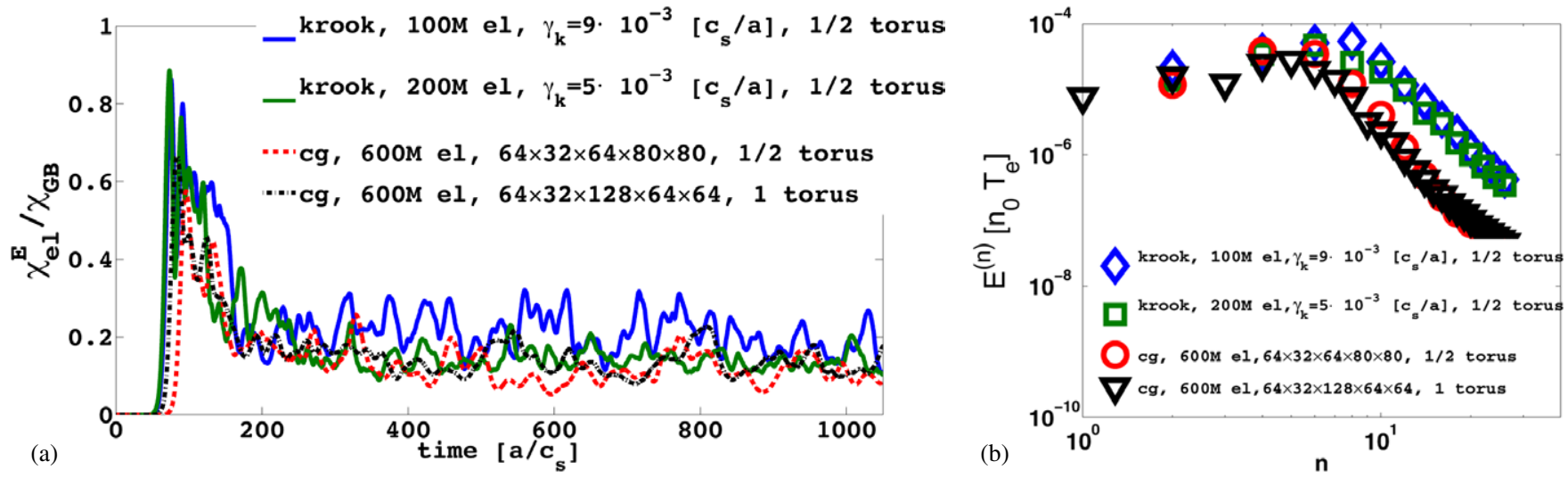

Figure 2. Small TEM CYCLONE case, $T_{\mathrm{i}}=T_{\mathrm{e}} / 3, \rho^{*}=1 / 60$. (a) Electron energy diffusivity $\chi_{\mathrm{el}}^{E}$ averaged between $r / a=0.2$ and $r / a=0.8$ compared for different noise-control methods, both Krook operator and coarse-graining (cg), and different numerical parameters. (b) Toroidal mode energy spectrum $E^{(n)}$ averaged between $t=800\left[a / c_{s}\right]$ and $t=1050\left[a / c_{s}\right]$. Differences at short wavelengths are observed between the coarse-graining procedure and the Krook operator.

figure 1. Only a very small difference is observed in the linear growth rates and real frequencies between local and global simulations. The weakness of finite- $\rho^{*}$ effects in linear temperature-gradient-driven TEM simulations is confirmed by ORB5 simulations presented in section 6.

\section{Comparison of noise-control methods}

In view of carrying out collisional, non-linear simulations it is essential to address the issue of numerical noise. In this section, a comparison of collisionless TEM simulations using different noise-control methods for electrons, either the Krook operator or the coarse-graining as explained in section 2.4, is performed within the frame of the hybrid model. The choice of the coefficient $\gamma_{\mathrm{K}}$ for the Krook operator, which needs to be significantly smaller (typically 10-20\%) than the maximum linear growth rate of the spectrum corresponding to the considered physical system, is naturally important, as well as the coarse-graining parameters. Note that, for a so far unknown reason, TEM simulations require a coarsegraining procedure applied to electrons at each time step in order to maintain a stationary transport level (while applying the coarse-graining every 10th time step is sufficient for ITG turbulence with adiabatic electrons [26]). At each time step, the electron $w$-weight is thus set to the average bin value, while the $p$-weight is unaffected. Moreover, a higher grid resolution in configuration space appears to be required for the coarsegraining binning in TEM simulations. As a consequence, a small plasma is easier to handle for the comparison between the Krook operator and the coarse-graining, due to the fact that a higher grid resolution in configuration space can be afforded. A reduced TEM CYCLONE case is thus chosen, considering $T_{\mathrm{i}}=T_{\mathrm{e}} / 3$ and $\rho^{*}=1 / 60$. Non-linear simulations are performed, using either the Krook operator or the coarsegraining (cg) procedure, for different numerical parameters as presented in figure 2( $a$ ) showing the electron energy diffusivity $\chi_{\mathrm{el}}^{E} / \chi_{\mathrm{GB}}$ averaged between $r / a=0.2$ and $r / a=0.8$. Within the statistical uncertainty range inherent to non-linear simulations, good agreement related to turbulent transport is obtained between the two noise-control methods. Table 1 summarizes the electron energy diffusivity $\chi_{\mathrm{el}}^{E} / \chi_{\mathrm{GB}}$ averaged between $t=800\left[a / c_{s}\right]$ and $t=1000\left[a / c_{s}\right]$ for the different cases considered in figure $2(a)$. However, as shown in figure $2(b)$ presenting the energy spectrum, the turbulent modes are more damped by the cg procedure in the range of shorter wavelengths $\left(n \sim 20\right.$ corresponding to $\left.k_{\theta} \rho_{s} \sim 1\right)$. Due to 
Table 1. Electron energy diffusivity $\chi_{\mathrm{el}}^{E} / \chi_{\mathrm{GB}}$ averaged between $r / a=0.2$ and $r / a=0.8$ as well as between $t=800\left[a / c_{s}\right]$ and $t=1000\left[a / c_{s}\right]$, for the four different cases presented in figure $2(a) .100 \mathrm{M}$ electron markers are probably not sufficient for a fully converged simulation considering the Krook operator. The Krook coefficients, $\gamma_{k}=9 \times 10^{-3}\left[c_{s} / a\right]$ and $\gamma_{k}=5 \times 10^{-3}\left[c_{s} / a\right]$, respectively, remain much smaller than the corresponding maximum linear growth rate $\gamma_{\max } \sim 0.18\left[c_{s} / a\right]$.

\begin{tabular}{lllll}
\hline & Krook, 100 M el. & Krook, 200 M el. & cg, $80 \times 80$ & cg, $64 \times 64$ \\
\hline$\chi_{\mathrm{el}}^{E} / \chi_{\mathrm{GB}}$ & 0.18 & 0.14 & 0.12 & 0.14 \\
\hline
\end{tabular}
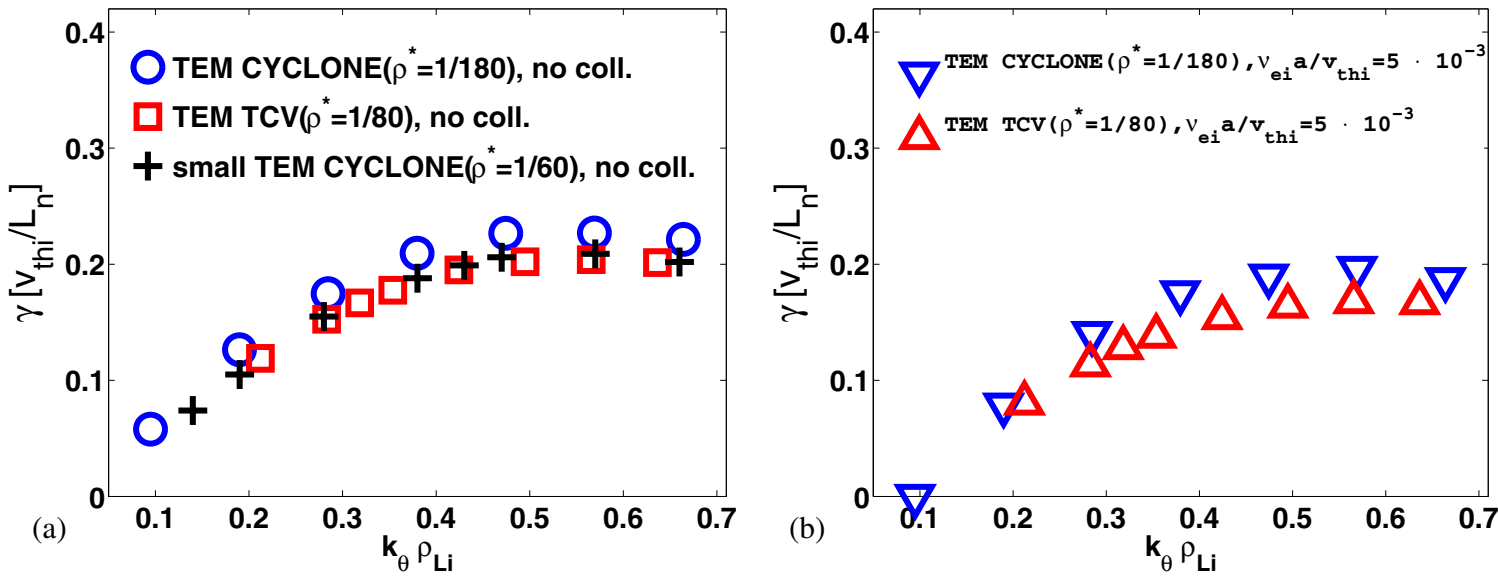

Figure 3. $k_{\theta}$-spectrum of the linear growth rate $\gamma$, for the TEM CYCLONE case $\left(\rho^{*}=1 / 180\right)$ and the TEM TCV case $\left(\rho^{*}=1 / 80\right)$ with $T_{\mathrm{e}}=T_{\mathrm{i}}$, considering $(a)$ a collisionless case and $(b)$ a collisional case. Additional collisionless results related to a small TEM CYCLONE case $\left(\rho^{*}=1 / 60, T_{\mathrm{e}}=T_{\mathrm{i}}\right.$ ) are shown as well. The influence of plasma size (through $\rho^{*}$ effects) on TEM linear growth rates turns out to be small, both for the collisionless and the collisional situations. As expected, electron collisions tend to reduce the linear growth rate at each value of $k_{\theta} \rho_{L \mathrm{i}}$

the limited role of short wavelength modes in the turbulent transport, the electron diffusivity is only marginally affected by the choice of the noise-control method, as already emphasized. Note however that, as already mentioned, the Krook operator is inappropriate for collisional simulations.

\section{Linear and non-linear $\rho^{*}$ effects in the TEM regime}

The plasma finite-size effects on the turbulence level, appearing through the $\rho^{*}=\rho_{s} / a$ parameter in global simulations, turn out to be important both in linear and nonlinear ITG regimes, as discussed e.g. in [15, 23, 32, 33]. Global simulations are found to provide lower linear growth rates and turbulent diffusivities than simulations performed in the local flux-tube limit $\left(\rho^{*} \rightarrow 0\right)$. As an illustration, let us quote results from the standard ITG-dominated CYCLONE case $\left(\rho^{*}=1 / 180, T_{\mathrm{i}}=T_{\mathrm{e}}, R / L_{T_{\mathrm{i}}}=R / L_{T_{\mathrm{e}}}=6.9, R / L_{n}=\right.$ 2.2). ORB5 CYCLONE simulations with adiabatic electrons provide a maximum linear growth rate $\gamma_{\max }\left(\rho^{*}=1 / 180\right) \simeq$ $0.12\left[v_{\text {thi }} / L_{n}\right]$, while the same simulations considering $\rho^{*}=$ $1 / 60$ give a significantly reduced maximum linear growth rate $\gamma_{\max }\left(\rho^{*}=1 / 60\right) \simeq 0.07\left[v_{\text {thi }} / L_{n}\right] \simeq 0.58 \gamma_{\max }\left(\rho^{*}=\right.$ $1 / 180)$. The situation in the TEM regime is however different. Figure $3(a)$ shows the $k_{\theta}$-spectrum of the collisionless TEM growth rate $\gamma$, for the TEM CYCLONE case $\left(\rho^{*}=1 / 180\right)$, the TEM TCV case $\left(\rho^{*}=1 / 80\right)$ and a small TEM CYCLONE case $\left(\rho^{*}=1 / 60\right)$, considering $T_{\mathrm{e}}=T_{\mathrm{i}}$ and the hybrid model for electrons. Peaked gradient profiles described by equation (22) with $\Delta_{\mathcal{A}}=0.2 a$ are used here. Although the
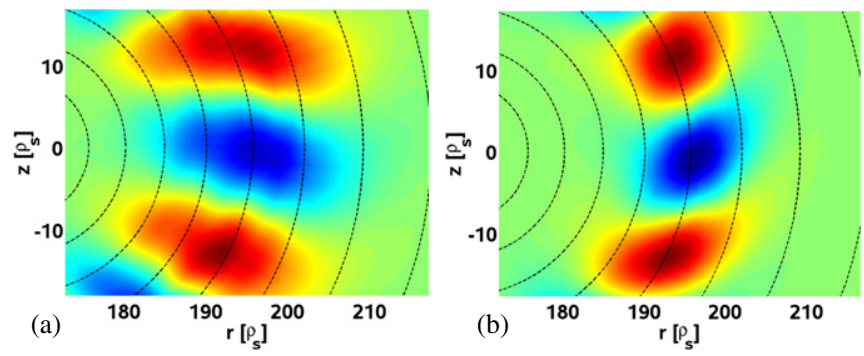

Figure 4. Linear structure of the mode $k_{\theta} \rho_{s}=0.3$, in the poloidal plane, for $(a)$ an ITG-dominated regime and $(b)$ a TEM-dominated regime in a small plasma $\left(\rho^{*}=1 / 60\right)$ considering $T_{\mathrm{e}}=T_{\mathrm{i}}$. TEM instabilities are more radially localized than ITG instabilities.

plasma size is different for each case, the linear growth rates appear to be only slightly reduced in a smaller plasma. This observation can be explained by the radial extension scaling of linear TEM modes. While the radial extension of ITG modes $\Delta r_{\text {ITG }}$ scales as $\Delta r_{\text {ITG }} / \rho_{s} \sim 1 / \sqrt{\rho^{*}}$ or $\Delta r_{\text {ITG }} / a \sim$ $\sqrt{\rho^{*}}$ [34-36], we observe that the radial extension of TEM modes $\Delta r_{\mathrm{TEM}}$ scales as $\Delta r_{\mathrm{TEM}} / \rho_{s} \sim 1 / \rho^{*}$ or $\Delta r_{\mathrm{TEM}} / a \sim$ constant. For the mode $k_{\theta} \rho_{s}=0.3$ corresponding to the TEM CYCLONE case, the radial extension $\Delta r_{\mathrm{TEM}} / a \simeq$ 0.2 is found for all $\rho^{*}$ values considered. The TEM modes are thus more radially localized in a small plasma, as illustrated in figure 4, leading to weaker profile shearing effects [37] due to finite plasma size and hence to a weaker reduction in growth rates compared with the ITG case. In addition, results of collisional linear simulations, accounting for both electron-ion and electron-electron collisions with 

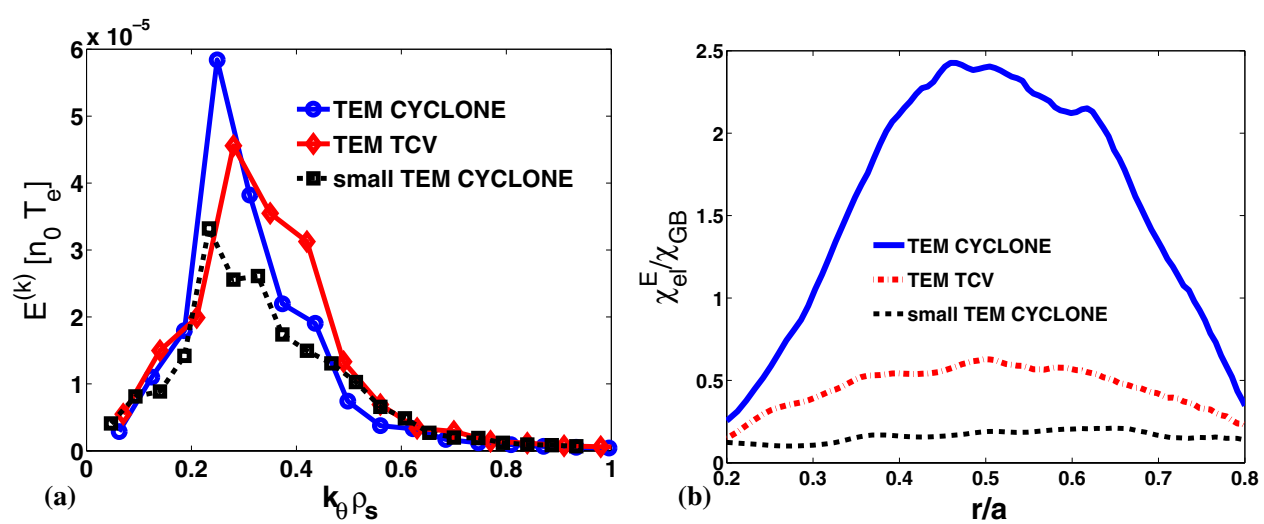

Figure 5. (a) Turbulence energy spectra averaged between $t=1000\left[a / c_{s}\right]$ and $t=1100\left[a / c_{s}\right]$ and $(b)$ electron energy diffusivity profile $\chi_{\mathrm{el}}^{E}(r)$ averaged between $t=800\left[a / c_{s}\right]$ and $t=950\left[a / c_{s}\right]$, for the TEM CYCLONE case $\left(\rho^{*}=1 / 180\right)$, the TEM TCV case $\left(\rho^{*}=1 / 80\right)$ and a small TEM CYCLONE case $\left(\rho^{*}=1 / 60\right)$, considering $T_{\mathrm{i}}=T_{\mathrm{e}} / 2$. Important non-linear finite- $\rho^{*}$-effects regarding the electron energy transport are observed, the diffusivity in gyro-Bohm units being significantly reduced in smaller plasmas (similarly to the ITG regime behaviour).

collisionality $v_{\mathrm{ei}} a / v_{\text {thi }}=5 \times 10^{-3}$, are shown in figure $3(b)$ and provide the same trend as the collisionless case regarding the plasma finite-size-effects, i.e. weak $\rho^{*}$ effects. Consistent with results obtained in section 9 , the linear growth rates are reduced by collisions through the electron detrapping process, regardless of the considered wavelength or plasma size. Note that, as mentioned in section 3, different temperature and density profile shapes are used for linear and non-linear simulations, which could provide different sensitivities to finite- $\rho^{*}$-effects. In order to address this issue and further pursue the study of finite- $\rho^{*}$-effects in linear TEM simulations, the TEM CYCLONE case is again considered, this time with $T_{\mathrm{i}}=T_{\mathrm{e}} / 3$ and using the wide gradient temperature and density profiles as given by equation (21), the same profiles as for nonlinear simulations. The growth rate of the mode $k_{\theta} \rho_{s}=0.5$ is found to be $\gamma\left(\rho^{*}=1 / 180\right) \simeq 0.27\left[c_{s} / L_{n}\right]$. The same simulation is repeated for $\rho^{*}=1 / 60$, i.e. corresponding to a small TEM CYCLONE case. The obtained growth rate, $\gamma\left(\rho^{*}=1 / 60\right) \simeq 0.24\left[c_{s} / L_{n}\right]$, is only marginally reduced $(\sim 10 \%)$ with respect to the TEM CYCLONE growth rate, which confirms the weak $\rho^{*}$ effects in linear temperaturegradient-driven TEM simulations.

Considering again the TEM CYCLONE case, the TEM TCV case and a small TEM CYCLONE case $\left(\rho^{*}=1 / 60\right)$, non-linear collisionless simulations of turbulence using the hybrid electron model are performed for $T_{\mathrm{i}}=T_{\mathrm{e}} / 2$ (the chosen temperature ratio aiming at stabilizing the ETG modes), and using the Krook operator in order to control the numerical noise $\left(\gamma_{k} \sim 0.03\left[c_{s} / a\right]\right)$. The turbulence energy spectra, averaged between $t=1000\left[a / c_{s}\right]$ and $t=1100\left[a / c_{s}\right]$, are similarly shaped but exhibit a turbulence level depending on the plasma size, as shown in figure 5(a). The electron energy diffusivity $\chi_{\mathrm{el}}^{E}$ in gyro-Bohm units, averaged between $t=800\left[a / c_{s}\right]$ and $t=950\left[a / c_{s}\right]$, is much larger for the largest plasma case, i.e. the TEM CYCLONE case, as presented in figure $5(b)$. The TEM-related turbulent electron transport reduction through finite- $\rho^{*}$-effects is thus of the same order as observed for ion transport in the ITG regime, i.e. a reduction in corresponding diffusivities by a factor of $\sim 4$ when going from $\rho^{*}=1 / 180$ to $\rho^{*}=1 / 80$ (see also figure 1 of [23]). As shown in figure $5(b)$, going from $\rho^{*}=1 / 180$ to $\rho^{*}=1 / 60$ reduces the electron energy diffusivity of the TEM CYCLONE case by a factor $\sim 10$ in gyro-Bohm units. In contrast to the $\rho^{*}$ effects in the linear TEM regime, the $\rho^{*}$ effects in the non-linear TEM regime thus appear to be important (regarding the turbulent transport), in agreement with [13].

\section{Effects of axisymmetric modes on temperature-gradient-driven TEM turbulence}

The axisymmetric electric fields, corresponding to the toroidal Fourier mode $n=0$, and associated zonal flows are a well-established mechanism for ITG turbulence saturation $[26,38]$. In the TEM regime, the effects of zonal flows on the saturation mechanism turn out to be less obvious. [8,9] claim that zonal flows do not contribute significantly to the TEM saturation mechanism for $\eta_{\mathrm{e}}=\nabla \ln T_{\mathrm{e}} / \nabla \ln n_{\mathrm{e}}>1$, i.e. for mainly temperature-gradient-driven TEM turbulence. This assertion is confirmed in [6], where only a limited role of zonal flows in TEM turbulence simulations is observed for the TEM CYCLONE case with both $T_{\mathrm{i}}=T_{\mathrm{e}}$ and $T_{\mathrm{i}}=T_{\mathrm{e}} / 3$. Here, additional confirmation regarding the latter conclusions is provided through new collisionless simulations (within the frame of the hybrid model) of the pure temperaturegradient-driven TEM turbulence case described in section 3, for which $\eta_{\mathrm{e}}=4.75$. Figure 6 shows the electron energy diffusivity profile $\chi_{\mathrm{el}}^{E}(r)$ averaged between $t=1000\left[a / c_{s}\right]$ and $t=1800\left[a / c_{s}\right]$, for both cases where the toroidal Fourier mode $n=0$ is retained or not in the simulation. The transport level is clearly only marginally affected by the presence of zonal flows, in contrast to the transport level in the ITG regime which is largely reduced in the presence of zonal flows. The Krook operator is used both for ions and electrons, with $\gamma_{k} \sim 0.01\left[c_{s} / a\right]$ significantly smaller than the maximum linear growth rate $\gamma_{\max } \sim 0.07\left[c_{s} / a\right]$ of the most unstable TEM.

\section{Varying the ETG in the TEM regime}

In this section, a comparison between linear and non-linear ETG scans is performed, considering the collisionless TEM 


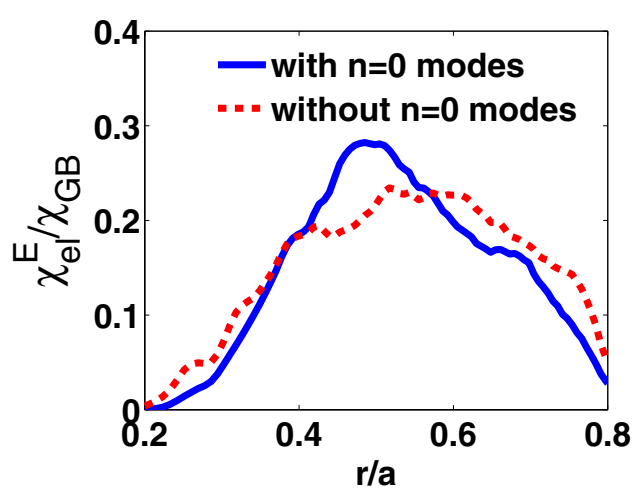

Figure 6. Effects of axisymmetric Fourier modes $(n=0, m)$ on electron transport level in the temperature-gradient-driven TEM regime (pure TEM case described in section 3). Electron energy diffusivity profile $\chi_{\mathrm{el}}^{E}(r)$ averaged between $t=1000\left[a / c_{s}\right]$ and $t=1800\left[a / c_{s}\right]$. Regarding the averaged turbulent transport level, no clear effect of zonal flows is visible in the temperature-gradient-driven TEM regime.

CYCLONE case with $T_{\mathrm{i}}=T_{\mathrm{e}} / 2$ along with the wide gradient density and temperature profiles (equation (21)). The Krook operator is used for controlling the numerical noise, both for electrons and ions. Note that the relaxation rate $\gamma_{k}$ does vary according to the maximum linear growth rate $\gamma_{\max }$ of the considered system, i.e. according to the ETG. An approximately constant ratio $\gamma_{k} / \gamma_{\max } \simeq 0.15$ is chosen throughout this section.

In the ITG regime, there is a well-established difference between the collisionless linear and non-linear critical ITGs, the so-called Dimits shift [29]: due to the shearing effects of zonal flows, a temperature gradient which provides a linear unstable ITG mode might be unable to sustain a nonvanishing turbulent transport if the corresponding maximum linear growth rate $\gamma_{\max }$ is of the order of the averaged $\vec{E} \times \vec{B}$ shearing rate $\left\langle\left|\omega_{E \times B}\right|\right\rangle_{r}$. For a finite turbulence level to persist, the ITG should provide a maximum growth rate $\gamma_{\max }$ that is clearly larger than the maximum possible zonal flow shearing rate, limited by Kelvin-Helmoltz-like instabilities: $\gamma_{\max }>\left\langle\left|\omega_{E \times B}\right|\right\rangle_{r}[26,39]$.

The situation in the temperature-gradient-driven TEM regime turns out to be different, as shown in figure 7 obtained with the TEM CYCLONE case parameters. Considering low ETGs $\left(a / L_{T \mathrm{e}} \lesssim 1.5\right)$, which provide marginally unstable linear TEM instabilities, a non-vanishing turbulent transport level is obtained in non-linear simulations. Both the linear growth rates $\gamma_{\max }$ and the non-linear turbulent diffusivities $\chi_{\mathrm{el}}^{E}$ appear to scale linearly with the ETG (the drive of turbulence) and to have approximately the same critical gradient $\left(a / L_{T_{\mathrm{e}}} \simeq 1\right)$, in contrast to the ITG regime where ITGs in the so-called Dimits shift region give rise to linear instabilities but cannot maintain a finite turbulent transport as a result of the shearing of turbulence by zonal flows [38]. Note that a finite level of electron energy transport is found even for ETGs at which $\gamma_{\max } \simeq\left\langle\left|\omega_{E \times B}\right|\right\rangle_{r}$, in contrast to the ITG regime situation. This illustrates the limited effects of zonal flows on temperature-gradient-driven TEM turbulence, as already discussed in section 7 .
A convergence check is performed for a particular case addressed in this section, i.e. considering the ETG $a / L_{T_{\mathrm{e}}}=$ 2.484. Increasing the electron marker number by a factor 2 , i.e. considering $200 \times 10^{6}$ electron markers, and reducing the relaxation rate by a factor 2 , i.e. considering $\gamma_{k} \simeq 0.015\left[c_{s} / a\right]$, provides an electron energy diffusivity of $\chi_{\mathrm{el}}^{E} / \chi_{\mathrm{GB}} \simeq 2.1$, averaged between $r / a=0.4$ and $r / a=0.6$. The latter value is within the typical uncertainty range of $15 \%$ [22] regarding the diffusivity $\chi_{\mathrm{el}}^{E} / \chi_{\mathrm{GB}} \simeq 2.35$, obtained with $100 \times 10^{6}$ electron markers and $\gamma_{k} \simeq 0.03\left[c_{s} / a\right]$ and shown in figure $7(b)$. Note that the signal/noise ratio, i.e. the energy of the nearly field-aligned Fourier modes divided by the energy of the unphysical, non-field-aligned Fourier modes, is satisfactory for both simulations (above 50). The latter result thus provides confidence as to the convergence of the presented TEM nonlinear results.

\section{Linear and non-linear collisional effects in the TEM regime}

While ion collisions tend to non-linearly increase the ITG turbulence through zonal flow damping [26,40], electron collisions have a strong and mostly linear effect on temperature-gradient-driven TEM instabilities, actually reducing the TEM drive through the electron detrapping process they generate. The most obvious collisional effect is thus the reduction in the TEM linear growth rates with increasing collisionality. The TEM TCV case is chosen for illustrating this latter fact, considering the following modified gradients: $a / L_{T_{\mathrm{i}}}=0, a / L_{T_{\mathrm{e}}}=1.70, a / L_{n}=0.85$ which provide an essentially pure TEM regime. Figure $8(a)$ shows the dependence on electron collisionality $v_{\mathrm{ei}}$ of the linear growth rate $\gamma$ corresponding to the mode $k_{\theta} \rho_{L \mathrm{i}}=0.4$, for different temperature ratios $T_{\mathrm{e}} / T_{\mathrm{i}}$ and collision operators. As expected, neglecting electron-electron collisions (electronion collisions only) leads to a weaker collisional growth rate reduction. Note that the collisional reduction in the growth rates is in accordance with numerical results obtained in [41] using the flux-tube version of the gyrokinetic code GEM. The collisionality $v_{\mathrm{ei}}$ is normalized with respect to the real frequency of the mode at zero collisionality $\omega\left(v_{\mathrm{ei}}=0\right)$, i.e. $\omega\left(v_{\mathrm{ei}}=0\right) \simeq 0.54\left[v_{\mathrm{thi}} / a\right]$ for $T_{\mathrm{i}}=T_{\mathrm{e}} / 3$ and $\omega\left(v_{\mathrm{ei}}=0\right) \simeq$ $0.38\left[v_{\text {thi }} / a\right]$ for $T_{\mathrm{i}}=T_{\mathrm{e}} / 2$. Note that $\omega$ remains essentially unaffected by electron collisions.

A small TEM CYCLONE case, i.e. the TEM CYCLONE case described in section 3 with $\rho^{*}=1 / 60$, is chosen in order to address the difference between linear and nonlinear collisional effects on temperature-gradient-driven TEM instabilities. The temperature ratio $T_{\mathrm{i}}=T_{\mathrm{e}} / 3$ is considered. The collisional reduction in the maximum linear growth rate $\gamma_{\max }$ (corresponding to the mode $k_{\theta} \rho_{L \mathrm{i}}=0.3$ ) with increasing electron-ion collision frequency $\nu_{\mathrm{ei}}$ is shown in figure $8(b)$ and compared with the collisional damping of the quasistationary electron turbulent energy flux $Q_{\mathrm{el}}$, averaged between $r / a=0.4$ and $r / a=0.6$. The reduction of non-linear turbulent transport (compared with a collisionless case) turns out to be stronger than the reduction in the linear growth rate and can thus not be explained by a simple mixing length 

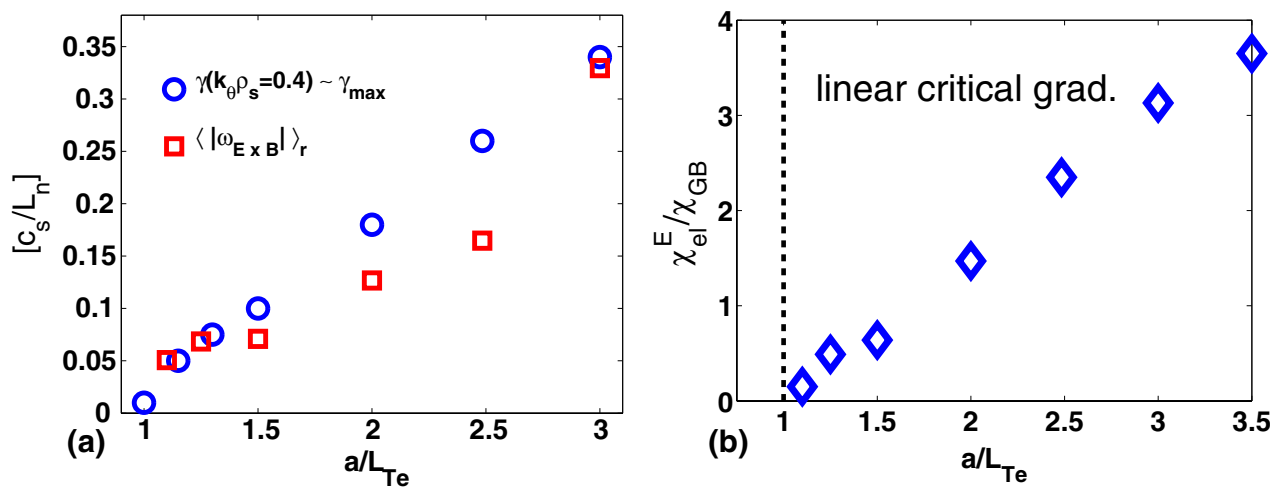

Figure 7. Electron temperature gradient $a / L_{T \mathrm{e}}$ dependence of (a) the maximum TEM linear growth rate $\gamma_{\max }$ and the radial average of the absolute value of the $\vec{E} \times \vec{B}$ shearing rate $\left\langle\left|\omega_{E \times B}\right|\right\rangle_{r}$ (averaged between $r / a=0.4$ and $r / a=0.6$ ), and (b) the electron energy diffusivity $\chi_{\mathrm{el}}^{E}$, averaged between $r / a=0.4$ and $r / a=0.6$ and over a time window of $\Delta t \sim 300\left[a / c_{s}\right]$, in the quasi-stationary state of the simulation $\left(t \sim 1000\left[a / c_{s}\right]\right)$. Linear and non-linear critical temperature gradients are obviously essentially identical $\left(a / L_{T_{\mathrm{e}}} \simeq 1\right)$.
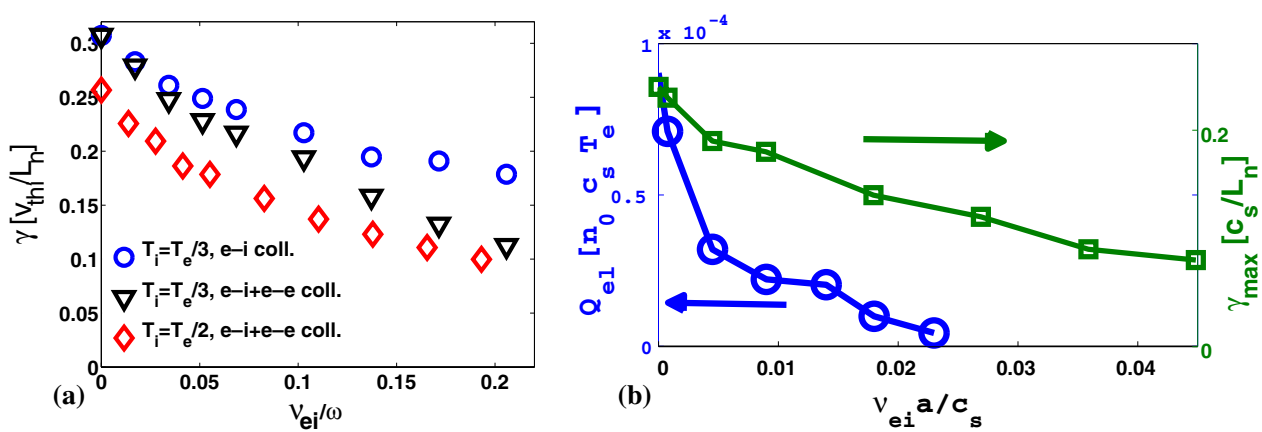

Figure 8. (a) Collisional damping of linear growth rates in the TEM regime. The TEM TCV case with $a / L_{T_{\mathrm{i}}}=0, a / L_{T_{\mathrm{e}}}=1.70$,

$a / L_{n}=0.85$, mode $k_{\theta} \rho_{L \mathrm{i}}=0.4$. The electron-ion collision frequency $v_{\mathrm{ei}}$ is normalized with respect to $\omega$, the collisionless real frequency of the considered mode $k_{\theta} \rho_{L \mathrm{i}}=0.4$. (b) The small TEM CYCLONE case, $T_{\mathrm{i}}=T_{\mathrm{e}} / 3$. Electron collisionality scan, both for the quasi-stationary electron turbulent energy flux $Q_{\mathrm{el}}$, averaged between $r / a=0.4$ and $r / a=0.6$, and for the maximum linear growth rate $\gamma_{\max }$. Electron collisions have a damping effect on TEM instabilities both in linear and non-linear simulations, the damping of non-linear turbulent transport (compared with a collisionless case) being however stronger than the damping of linear growth rates.

argument $\chi \sim \gamma_{\max } /\left\langle k_{\perp}^{2}\right\rangle$. Note that the real frequency of the mode $k_{\theta} \rho_{L \mathrm{i}}=0.3, \omega \simeq 0.48\left[c_{s} / L_{n}\right]$, remains essentially unaffected by collisions, and that both electronion and electron-electron collisions are accounted for. The non-linear runs for simulating collisional TEM turbulence presented in figure $8(b)$ are actually very challenging due to the large number of markers required $\left(\sim 600 \times 10^{6}\right.$ electrons and $\sim 200 \times 10^{6}$ ions) combined with the small time step related to electron dynamics, leading to $\sim 500000 \mathrm{CPU}$ hours consumed for each simulation.

Considering again the small TEM CYCLONE case with $T_{\mathrm{i}}=T_{\mathrm{e}} / 3$, figure 9 shows the turbulent component (in the poloidal plane) of the perturbed electrostatic potential, $e\left(\phi-\langle\phi\rangle_{S}\right) / T_{\mathrm{e} 0}$, at $t=1000\left[a / c_{s}\right]$ for the two collisionalities $\nu_{\mathrm{ei}} a / c_{s}(r / a=0.5)=7.5 \times 10^{-4}$ and $v_{\mathrm{ei}} a / c_{s}(r / a=$ $0.5)=4.5 \times 10^{-3}$. As expected, the averaged turbulence level is weaker for a stronger collisionality. Note that the collisionality dependence on density and temperature profiles is accounted for in ORB5 simulations, leading in fact to stronger collisionality close to the plasma edge and weaker collisionality close to the magnetic axis. The collisionality increase towards the edge is explained by a weaker reduction in collisionality due to lower density $(\sim n)$ than the increase of collisionality due to lower temperature $\left(\sim T_{\mathrm{e}}^{-3 / 2}\right)$. Compared with the weakly collisional situation, the TEM turbulence in the case of stronger collisionality is thus more reduced and even quenched when going towards the plasma edge as illustrated in figure 9.

\section{Conclusions}

In this paper, results from global gyrokinetic simulations of temperature-gradient-driven TEM turbulence performed with the ORB5 code are discussed. Two different methods for controlling the numerical noise (inherent to the PIC approach) are evaluated and compared. A linear benchmark in the TEM regime against the Eulerian gyrokinetic code GENE is presented (accounting for fully kinetic electron response), which shows good agreement between both codes. The GENE-ORB5 comparison, however, points out the limitations of the polarization drift term in the quasi-neutrality equation considered only to second order in $k_{\perp} \rho_{L}$ in ORB5. While the long wavelength approximation is valid for computing electron heat fluxes with the parameters considered in this paper, it is nonetheless a limiting factor of the ORB5 code if one intends to explore a larger range of parameters and is therefore currently being addressed through the implementation of an integral 

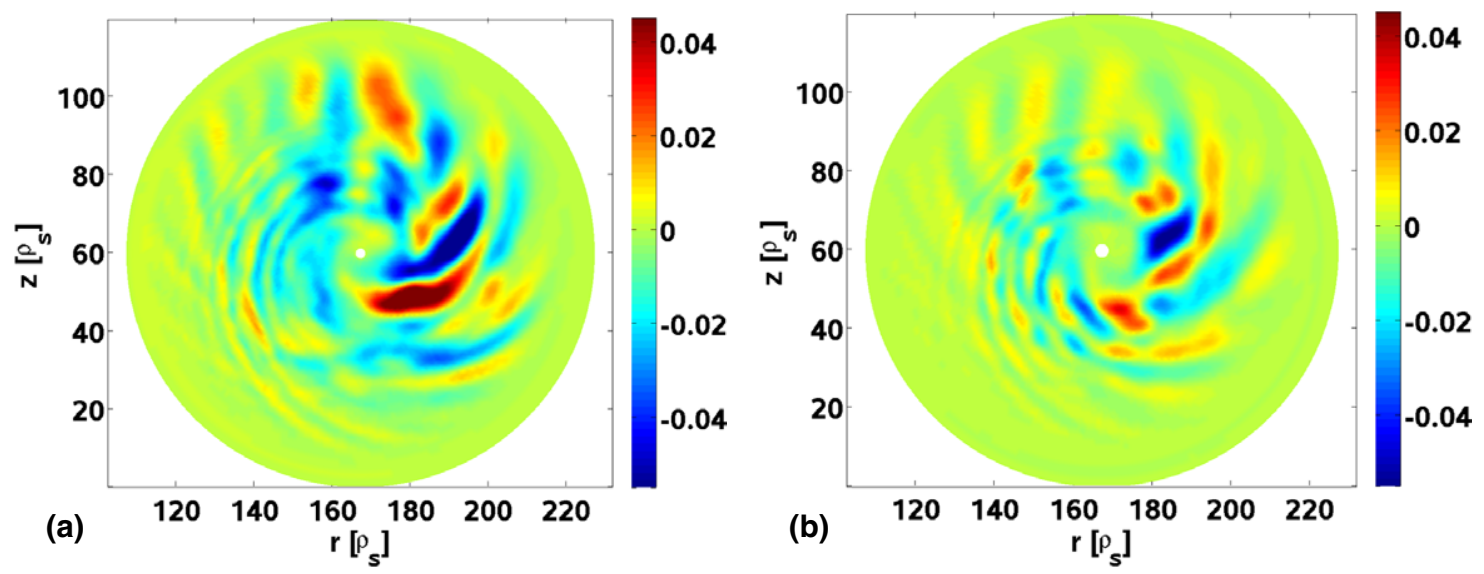

Figure 9. Small TEM CYCLONE case, $T_{\mathrm{i}}=T_{\mathrm{e}} / 3$. Poloidal cross-section of the non-zonal component of the electrostatic potential $e\left(\phi-\langle\phi\rangle_{S}\right) / T_{\mathrm{e} 0}$ at $t=1000\left[a / c_{s}\right]$ for the two collisionalities $(a) v_{\mathrm{ei}} a / c_{s}(r / a=0.5)=7.5 \times 10^{-4}$ and (b) $\nu_{\mathrm{ei}} a / c_{s}(r / a=0.5)=4.5 \times 10^{-3}$. The turbulence reduction through electron collisions is clearly visible.

form of the polarization drift operator valid to all orders in $k_{\perp} \rho_{L}$ [42].

The effects of finite plasma size on TEM instabilities turn out to be important in the non-linear regime, while there is almost no effect of finite plasma size in the TEM linear regime. Note the difference compared with ITG-dominated cases, where the effects of finite plasma size are important both in linear and non-linear regimes.

In the temperature-gradient-driven TEM regime, the zonal flows are shown to play no role in turbulence saturation, confirming previous studies. Accordingly, no Dimits shift region is observed in this regime, unlike in the ITG regime that features a Dimits shift region as a consequence of the importance of zonal flow shearing in turbulence saturation.

Electron collisions are found to reduce both the linear growth rate and the transport level related to TEM instabilities, through the electron detrapping process. Note that, in contrast, the ion collision effects on ITG turbulence are almost fully nonlinear and lead to a general increase in ITG turbulence level through zonal flow damping $[26,40]$.

\section{Acknowledgments}

Simulations were performed on the Monte Rosa CRAY XE-6 supercomputer of the Swiss National Supercomputing Center, the HPC-FF cluster of the Jülich Forschungszentrum, the BlueGene/P supercomputer of the CADMOS project and the IFERC-CSC HELIOS platform. This work was partly supported by the Swiss National Science Foundation.

(C) Euratom 2013.

\section{References}

[1] Falchetto G L, Vaclavik J and Villard L 2003 Phys. Plasmas $101424-36$

[2] Waltz R E, Austin M E, Burrell K H and Candy J 2006 Phys. Plasmas 13052301

[3] Chowdhury J, Ganesh R, Angelino P, Vaclavik J, Villard L and Brunner S 2008 Phys. Plasmas 15072117
[4] Bottino A, Peeters A G, Sauter O, Vaclavik J, Villard L and Team A U (ASDEX Upgrade Team) 2004 Phys. Plasmas 11 198-206

[5] Bottino A, Sauter O, Camenen Y and Fable E 2006 Plasma Phys. Control. Fusion 48215

[6] Jolliet S 2009 Gyrokinetic particle-in-cell global simulations of ion-temperature-gradient and collisionless-trapped-electron-mode turbulence in tokamaks PhD Thesis Ecole polytechnique fédérale de Lausanne

[7] Lee W 1987 J. Comput. Phys. 72 243-69

[8] Dannert T and Jenko F 2005 Phys. Plasmas 12072309

[9] Ernst D R, Lang J, Nevins W M, Hoffman M, Chen Y, Dorland W and Parker S 2009 Phys. Plasmas 16055906

[10] Xiao Y, Holod I, Zhang W, Klasky S and Lin Z 2010 Phys. Plasmas 17022302

[11] Lang J, Parker S E and Chen Y 2008 Phys. Plasmas 15055907

[12] Merz F and Jenko F 2008 Phys. Rev. Lett. 100035005

[13] Xiao Y and Lin Z 2009 Phys. Rev. Lett. 103085004

[14] Scott B and Smirnov J 2010 Phys. Plasmas 17112302

[15] Görler T, Lapillonne X, Brunner S, Dannert T, Jenko F, Merz F and Told D 2011 J. Comput. Phys. 230 7053-71

[16] Tran T M, Appert K, Fivaz M, Jost G, Vaclavik J and Villard L 1999 Theory of Fusion Plasmas, Int. Workshop (Bologna Editrice Compositori, Società Italiana di Fisica) p 45

[17] Jolliet S, Bottino A, Angelino P, Hatzky R, Tran T, McMillan B, Sauter O, Appert K, Idomura Y and Villard L 2007 Comput. Phys. Commun. 177 409-25

[18] Lin Z, Tang W M and Lee W W 1995 Phys. Plasmas 2 2975-88

[19] Brunner S, Valeo E and Krommes J A 1999 Phys. Plasmas 6 4504-21

[20] Vernay T, Brunner S, Villard L, McMillan B F, Jolliet S, Tran T M, Bottino A and Graves J P 2010 Phys. Plasmas 17122301

[21] Hahm T S 1988 Phys. Fluids 31 2670-3

[22] McMillan B F, Jolliet S, Tran T M, Villard L, Bottino A and Angelino P 2008 Phys. Plasmas 15052308

[23] McMillan B F, Lapillonne X, Brunner S, Villard L, Jolliet S, Bottino A, Görler T and Jenko F 2010 Phys. Rev. Lett. 105155001

[24] Lapillonne X, McMillan B F, Görler T, Brunner S, Dannert T, Jenko F, Merz F and Villard L 2010 Phys. Plasmas 17112321

[25] Chen Y and Parker S E 2007 Phys. Plasmas 14082301

[26] Vernay T, Brunner S, Villard L, McMillan B F, Jolliet S, Tran T M and Bottino A 2012 Phys. Plasmas 19042301 
[27] Lapillonne X, Brunner S, Dannert T, Jolliet S, Marinoni A, Villard L, Görler T, Jenko F and Merz F 2009 Phys. Plasmas 16032308

[28] Hahm T S 1994 Phys. Plasmas 1 2940-2944

[29] Dimits A M et al 2000 Phys. Plasmas 7 969-983

[30] Bottino A 2004 Modelling of global electrostatic microinstabilities in tokamaks: effects of $E \times B$ flow and magnetic shear $P h D$ Thesis Ecole polytechnique fédérale de Lausanne

[31] Merz F and Jenko F 2010 Nucl. Fusion 50054005

[32] Lin Z, Ethier S, Hahm T S and Tang W M 2002 Phys. Rev. Lett. 88195004

[33] Candy J, Waltz R E and Dorland W 2004 Phys. Plasmas 11 L25-8

[34] Romanelli F and Zonca F 1993 Phys. Fluids B: Plasma Phys. 5 4081-9
[35] Brunner S, Fivaz M, Tran T M and Vaclavik J 1998 Phys. Plasmas 5 3929-49

[36] Garbet X, Idomura Y, Villard L and Watanabe T 2010 Nucl. Fusion $\mathbf{5 0} 043002$

[37] Waltz R E, Candy J M and Rosenbluth M N 2002 Phys. Plasmas 9 1938-46

[38] Lin Z, Hahm T S, Lee W W, Tang W M and White R B 1998 Science 281 1835-7

[39] Waltz R E, Dewar R L and Garbet X 1998 Phys. Plasmas 5 1784-92

[40] Lin Z, Hahm T S, Lee W W, Tang W M and Diamond P H 1999 Phys. Rev. Lett. 83 3645-8

[41] Lang J, Chen Y and Parker S E 2007 Phys. Plasmas 14082315

[42] Mishchenko A, Könies A and Hatzky R 2005 Phys. Plasmas 12062305 